Manuscript version of the paper published in Astrophysics and Space Science

\title{
Some Clues to Understand MOND and Accelerated Expansion of the Universe
}

\author{
Hasmukh K. Tank \\ Indian Space Research Organization \\ 22/693, Krishna Dham-2, Vejalpur, Ahmedabad 380015, India \\ E-mail: hasmukh.tank1@gmail.com
}

\begin{abstract}
This letter points out that the values of 'critical-acceleration' of MOND, and the 'accelerated-expansion' of the universe are just two of the fourteen strikingly equal values of accelerations recurring in different physical situations. Alternative expressions for the cosmological red-shift, the 'critical-acceleration' of MOND and Newton's law of universal gravitation are also presented.
\end{abstract}

Key Words Gravitation, Cosmology, Cosmological red-shift, Gravitational red-shift, Dark-matter, Dark-energy, MOND, Acceleratedexpansion of the universe

\section{Introduction}

Two major conundrums have arisen in astrophysics and cosmology in recent times. The first was the realization that there are not enough visible stars (or gas) inside galaxies to account for their high rate of rotation. The theory of dark matter was created to explain this phenomenon. It theorizes that the galaxies are spinning as fast as they are because there is more matter in those galaxies (including our own Milky Way) than can be seen by counting the mass of stars and gas alone; and that this unseen (dark) matter is invisible because it does not interact with light (i. e. the electromagnetic force) which we use to see things.

An alternative explanation for the galaxies' rotation-curves was proposed by Milgrom. According to his Modified Newtonian Dynamics [MOND] the actual value of acceleration $a$ experienced by the stars in the galaxies is not:

$a=G M / r^{2}$ as Newton's theory would predict; rather it is:
$a=\left[G M a_{0}\right]^{1 / 2} / r$

(where $a_{0}$ is a new constant of nature $a_{0}=1.2 \mathrm{x}$ $10^{-10} \mathrm{~m} / \mathrm{s}$ )

To explain the meaning of this constant, Milgrom said: "... It is roughly the acceleration that will take an object from rest to the speed of light in the lifetime of the universe. It is also of the order of the recently discovered acceleration of the universe."

Recently, Gupta and Pradhan have presented a theoretically explained new variant of MOND (Gupta R. C. and Pradhan Anirudh, Astrophysics and Space Science, Vol. 333, 2011), raising the status of MOND from 'phenomenological' to 'theoretically understandable' theory; and their subsequent paper presents a unified approach to MOND and 'the accelerated expansion of the universe' (Gupta, R. C. and Pradhan Anirudh, arXiv.org/abs/1010.3826, October 2011).

The second conundrum came in 1998, that the distant supernovae were fainter, and thus farther away than they expected. . The observations of Type Ia supernovae suggested that the universe was not only expanding, but also accelerating its expansion. (Riess, A. et al. 1998 and Perlmutter, S. et al. 1999) In the past few years, these observations have been corroborated by several independent sources: the cosmic microwave background radiation and large scale structure (Spergel, D. N., et al. 2003), age of the universe (Chaboyer, B., \& Krauss, L. M. 2002) as well as improved measurements of the supernova (WoodVasey, W. M., et al. 2007 and Astier, P., et al. 2006) and X-ray properties of galaxy clusters. Currently popular theories attempting to explain accelerating expansion include some form of dark energy: Cosmological Constant, Quintessence, Dark Fluid or Phantom energy. The most important 
property of dark energy is that it has negative pressure which is distributed relatively homogeneously in space.

This letter points out an interesting coincidence, that the values of 'critical-acceleration' of MOND, and the 'accelerated-expansion' of the universe are just two of the fourteen strikingly equal values of accelerations recurring in different physical situations, as shown below.

\section{Recurrences of the same value of acceleration $H_{0} c$ in fourteen different physical situations}

The carefully-measured values of decelerations of Pioneer-10, Pioneer-11, Galileo and Ulysses spaceprobes (Anderson, J.D., et. al. 1998) show that they are all almost equal. First of all, the matching of accelerations of four different space-probes, in spite of their different masses, speeds and directions, is itself a striking observation; and their matching with the deceleration of cosmologicallyred-shifting-photons (Tank H. K. 2010), the 'critical-acceleration' of MOND and the 'accelerated-expansion' of the universe, can not be ignored by a scientific mind as an accidental coincidence. Perfect matching of seven different phenomena can not be an accident. In addition to that, Sivaram has noticed that (Sivaram, 1994):

$$
\begin{aligned}
& G M_{0} / R_{0}^{2}=G m_{\mathrm{P}} / r_{\mathrm{P}}^{2}=G m_{\mathrm{e}} / r_{\mathrm{e}}^{2}=G m_{\mathrm{n}} / r_{\mathrm{n}}^{2} \\
& =G M_{\mathrm{gc}} / R_{\mathrm{gc}}{ }^{2}=G M_{\mathrm{gal}} / R_{\mathrm{gal}}{ }^{2}=G M_{\mathrm{cg}} / R_{\mathrm{cg}}{ }^{2} \\
& =H_{0} c
\end{aligned}
$$$$
=\text { The 'critical-acceleration' of MOND }
$$

( Here: $\quad M_{0}$ and $R_{0}$ are mass and radius of the universe respectively, $m_{\mathrm{P}}$ and $r_{\mathrm{P}}$ are mass and radius of the proton, $m_{\mathrm{e}}$ and $r_{\mathrm{e}}$ are mass and radius of the electron, $m_{\mathrm{n}}$ and $r_{\mathrm{n}}$ are mass and radius of the nucleus of an atom, $M_{\mathrm{gc}}$ and $R_{\mathrm{gc}}$ are mass and radius of the globular-clusters, $M_{\text {gal }}$ and $R_{\text {gal }}$ are mass and radius of the spiral-galaxies, and $M_{\mathrm{cg}}$ and $R_{\mathrm{cg}}$ are mass and radius of the galactic-clusters respectively.)

Thus, the value of 'accelerated-expansion' of the universe and the 'critical-acceleration' of MOND are just two of the fourteen strikingly matching values of accelerations of different physical phenomena, whose details are as follows:

The carefully measured values of accelerations experienced by the space-probes Pioneer-10, Pioneer-11, Galileo, and Ulysses are (Anderson, J.D., et. al. 1998):

For Pioneer-10, $a=(8.09 \pm 0.2) \times 10^{-10} \mathrm{~m} / \mathrm{s}^{2}$,

For Pioneer-11, $a=(8.56 \pm 0.15) \times 10^{-10} \mathrm{~m} / \mathrm{s}^{2}$,

For Ulysses, $\quad a=(12 \pm 3) \times 10^{-10} \mathrm{~m} / \mathrm{s}^{2}$,

For Galileo, $\quad a=(8.0 \pm 3) \times 10^{-10} \mathrm{~m} / \mathrm{s}^{2}$,

For the cosmologically-red-shifted-photons:

$a=6.87 \times 10^{-10} \mathrm{~meter} / \mathrm{sec}^{2}=H_{0} c$ as shown below:

\section{The cosmological-red-shift smaller than unity} can also be expressed as deceleration experienced by the photons (Tank H. K. 2010):

The cosmological red shift,

$z_{\mathrm{c}}=\Delta f / f=\left(f_{0}-f\right) / f=H_{0} D / c$.

(Where: $f_{0}$ is frequency of light emitted by a distant star; $f$ is frequency of light received on the earth; $H_{0}$ is Hubble's constant; $D$ is luminosity-distance; and $c$ is the speed of light in vacuum.)

We can write the expression-2 as:

$$
h \Delta f / h f=H_{0} D / c \text {, }
$$

(Where, $h$ is Planck's constant; so that $h \Delta f$ and $h$ $f$ have the dimension of energy)

i.e. $\quad h \Delta f=\left(h f / c^{2}\right)\left(\underline{H}_{\underline{O}} c\right) D$

That is, the loss in energy of the photon $(h \Delta f)$ is equal to: its mass $\left(h f / c^{2}\right)$ times acceleration $\left(H_{0} \underline{c}\right)$ times the luminosity-distance $(D)$.

In the expression-3, we find that the deceleration $\left(H_{0} c\right)$ experienced by the cosmologically-redshifting-photon is also equal to the decelerations observed in the case of Pioneer-10, Pioneer-11, Galileo and Ulysses spacecrafts, and all these decelerations match strikingly with the "critical 
acceleration' of MOND, and 'accelerated expansion' of the universe. Perfect matching of accelerations of fourteen different phenomena can not be an accidental-coincidence. In this universe, of huge mass and very large radius, the spaceprobes: Pioneer-10, 11, Galileo and Ulysses are as good as photons; so they too experience similar amount of acceleration.

\section{The flattening of galaxies' rotation-curves can also be expressed as:}

The constant-velocity of the stars at the out-skirts of galaxies, $v=\left[M / M_{0}\right]^{1 / 4} c$

(Where: $M$ is mass of the galaxy, $M_{0}$ is total-mass of the universe, and $c$ is the speed of light)

\section{Newton's law of universal gravitation can also be expressed as:}

The gravitational-potential-energy of a system of masses $M$ and $m$ is:

$G M m / r=\left[M / M_{0}\right]\left(m c^{2}\right) /\left[r / R_{0}\right]$....(7)

\section{References}

1. Anderson, J.D., Laing, P.A., Lau, E.L., Liu, A.S., Nieto M. M., and Turyshev. S.G. Indication, from Pioneer 10/11, Galileo, and Ulysses Data, of an Apparent Anomalous, Weak, Long-Range Acceleration, Phys.Rev. Letters 81 (1998) 28582861 [Comment by Katz J.I.: Phys. Rev. Lett. 83, 1892 (1998)]

2. Astier, P., et al. The Supernova Legacy Survey: measurement of $\Omega_{\mathrm{M}}, \Omega_{\mathrm{X}}$ and $w$ from the first year data set, 2006, Astronomy and Astrophysics, 447, 31

3. Chaboyer, B., \& Krauss, L. M. Observational constraints on the brane-world model with branebulk energy exchange , 2002, Astrophysical Journal Letters, 567, L4

4. Gupta, R. C. and Pradhan, Anirudh, 'A Theoretically-explained New-variant of Modified Newtonian Dynamics (MOND)', Int. J. of Astrophysics Space Science, Springer-publication, 333, pp.311-316, Jan/Feb 2011. 5. R.C. Gupta, and Anirudh Pradhan, 'Is Hubble's Expansion due to Dark Energy', www.arxiv.org/abs/1010.3826, Oct. 2010.
6. Perlmutter, S. et al. Measurements of $\Omega$ and $\Lambda$ from 42 High-Redshift Supernovae 1999, Astrophysical Journal, 517, 565

7. Reiss A. et al. Observational evidence from supernovae for an accelerating universe and a cosmological constant, 1998, Astronomical Journal, 116, 1009

8. Sivaram C. Some aspects of MOND and its consequences for cosmology, Astrophysics and Space Science, 1994, 215, 185-189. .

9. Spergel, D. N., et al. First-Year Wilkinson Microwave Anisotropy Probe (WMAP)* Observations: Determination of Cosmological Parameters 2003, Astrophysical Journal Supplement, 148, 175

10. Tank, H. K. A new law emerging from the recurrences of the 'critical-acceleration' of MOND, suggesting a clue to unification of fundamental forces, Astrophysics and Space Science, 2010, 330, 203-205

11. Tank, H. K. Wave-theoretical explanation for the newly-emerged-law of equality of potentialenergy and energy-of-mass of reasonably independent systems of matter, Advanced Studies in Theoretical Physics, 2011, 5, 45-55.

12.Wood-Vasey, W. M., et al. Observational Constraints on the Nature of the Dark Energy: First Cosmological Results from the ESSENCE Supernova Survey, 2007, Astrophysical Journal, 666, 694 